\title{
The Influence of the Substitution Rate of Mixed Material in Green Concrete
}

\author{
Liu Wenyan, Meng Yubing, Lu Yue, Wang Zhenlin, Wang Yanzeng \\ Shandong Zhongjian Western Construction Co. LTD., Qingdao, China
}

Email address:

404152198@QQ.com (Meng Yubing)

To cite this article:

Liu Wenyan, Meng Yubing, Lu Yue, Wang Zhenlin, Wang Yanzeng. The Influence of the Substitution Rate of Mixed Material in Green Concrete. Science Discovery. Vol. 6, No. 4, 2018, pp. 224-230. doi: 10.11648/j.sd.20180604.11

Received: May 2, 2018; Accepted: June 26, 2018; Published: July 20, 2018

\begin{abstract}
In order to meet the needs of long-term sustainable development of the country, concrete greening has become more and more important in the environment of energy conservation and emission reduction and environmental protection. The use of concrete admixtures can greatly reduce the amount of cement, and it can improve the concrete's flexibility and improve the long-term performance of concrete. As the most common combination of mineral powder and fly ash, both of them can be used to complement each other in concrete, and make full use of energy efficiency to improve concrete performance and reduce production cost. How to better play the role of the mineral powder and fly ash is the problem that the concrete enterprise ADAPTS to The Times development to meet the market competition and needs the active research.
\end{abstract}

Keywords: Blending Material, Flow Degree, Mortar Strength, Substitution Rate

\section{绿色混凝土中掺合料取代率的影响}

刘文燕, 孟玉冰, 卢月, 王振林, 王延增

山东中建西部建设有限公司，青岛，中国

邮箱

404152198@QQ.com（孟玉冰）

摘要：为适应国家长期可持续发展的需要, 在节能减排、保护环境的大环境下, 混凝土绿色化已经越来越重要。混凝 土掺合料的使用可以大幅度降低水泥的用量, 而且可以改善混凝土的和易性, 提升混凝土的长期性能。作为最常见的 掺合料矿粉和粉煤灰, 二者同时利用能够在混凝土中优势互补, 充分发挥能效提高混凝土性能, 降低生产成本。如何 更好的发挥矿粉和粉煤灰的作用是混凝土企业适应时代发展满足市场竞争, 需要积极研究的问题。本文通过矿粉和粉 煤灰多种取代率的排列组合, 对胶砂的流动度、 $7 \mathrm{~d} 28 \mathrm{~d}$ 抗折强度、 $7 \mathrm{~d} 28 \mathrm{~d}$ 抗压强度展开研究, 通过对比试验求得经济高 效并满足混凝土使用要求的取代率组合。研究表明掺合料的使用可以明显改善胶砂流动性, 随着掺合料的使用胶砂强 度呈现先增后降的趋势, 并且矿粉的掺量不影响粉煤灰性能的发挥。

关键词：绿色混凝土，掺合料，流动度，胶砂强度，取代率 


\section{1. 引言}

随着经济的飞速发展, 资源和能源供应越来越紧张, 同时也加重了环境恶化, 形成经济发展的瓶颈。混凝土 作为当今世界上建筑工程中使用量最大、使用范围最广 的工程材料, 研究、开发和应用绿色混凝土已经成为一 种趋势。由于对水泥需求的不断增长导致水泥生产企业 大量煅烧熟料，水泥工业排放的 $\mathrm{CO}_{2}$ 量占到总排放量的 1/10左右, 是全球“温室气体”的重要源头, 水泥生产还需 要大量化石燃料, 排放大量 $\mathrm{CO} 、 \mathrm{SO} 2$, 和 $\mathrm{NO}_{\mathrm{X}}$ 等有害气 体。混凝土材料的生产和使用消耗了大量的能用和资源, 给人类的生存环境带来巨大的负担。温室气体、有毒有 害气体、粉尘等被大量排放到大气中, 而绿色混凝土大 量合理的提高掺合料使用量, 降低水泥用量, 减少污染 排放降低能耗, 将粉煤灰等工业垃圾变废为宝, 还可以 使混凝土易于浇筑、捣实而不离析, 改善混凝土耐久性、 和易性, 降低水化峰值、增加体积稳定性, 并长期保持 良好的力学性能。

当前研究多分别针对单一掺合料进行, 而实际使用中 多为双掺甚至多掺。本文将通过对混凝土活性掺合料的复 合使用, 研究其对胶砂流动性、抗折性能、抗压性能的影 响, 以达到改善混凝土性能、减少水泥使用量的目的, 对 降低碳排放、改善生态环境更是具有深远的意义。

\section{2. 试验安排}

当前配合比设计中使用最多的矿物掺合料是矿渣粉 和粉煤灰, 其使用量或使用比例是按照标准JGJ55-2011 《普通混凝土配合比设计规程》设计的, 其中 3.0 .5 条规定 矿物掺合料在混凝土中的掺量应通过试验确定, 并规定了 最大掺量。现通过胶砂试验探究掺合料取代率不同对胶砂 强度、流动度的影响, 从而进一步探讨不同的掺合料取代 率对混凝土强度、流动度的影响。

\section{1. 试验设计}

为了探究掺合料取代水泥后对胶砂强度、流动度的影 响, 本方案将对不同取代率的掺合料+水泥进行胶砂试验, 以纯水泥试验状态为基准, 结合 《普通混凝土配合比设计规 程》中矿物掺合料的最大掺量规定, 此次试验掺合料取代率 最高为 $50 \%$, 分别进行以下胶砂试验对比: 单掺矿粉 $10 \%$ 、 $20 \% 、 30 \%$ ，单掺粉煤灰 $10 \% 、 20 \% 、 30 \%$ ，双掺粉煤灰 $10 \%+$ 矿粉 $10 \%$ 、粉煤灰 $10 \%+$ 矿粉 $20 \%$ 、粉煤灰 $10 \%$ +矿粉 $30 \%$ 、 粉煤灰 $10 \%$ +矿粉 $40 \%$ 、粉煤灰 $20 \%+$ 矿粉 $10 \%$ 、粉煤灰 $20 \%+$ 矿粉 $20 \%$ 、粉煤灰 $20 \%+$ 矿粉 $30 \%$ 、粉煤灰 $30 \%$ +矿粉 $10 \%$ 、 粉煤灰 $30 \%+$ 矿粉 $20 \%$ 、粉煤灰 $40 \%+$ +矿粉 $10 \%$ ，通过对以上 17 种组合的胶砂流动度、强度的检测, 来探究掺合料取代率 对胶砂流动度、强度的影响, 寻找最佳取代率。

表1 材料用量。

\begin{tabular}{|c|c|c|c|c|c|}
\hline 胶砂 & 水泥用量 $(\mathrm{g})$ & 矿粉用量 (g) & 粉煤灰用量（g） & 标准砂用量 (g) & 用水量 $(\mathrm{ml})$ \\
\hline 水泥 & 450 & 0 & 0 & 1350 & 225 \\
\hline 矿粉 $10 \%$ & 405 & 45 & 0 & 1350 & 225 \\
\hline 粉煤灰 $10 \%$ & 405 & 0 & 45 & 1350 & 225 \\
\hline 矿粉 $20 \%$ & 360 & 90 & 0 & 1350 & 225 \\
\hline $10 \%$ 矿粉, $10 \%$ 粉煤灰 & 360 & 45 & 45 & 1350 & 225 \\
\hline 粉煤灰20\% & 360 & 0 & 90 & 1350 & 225 \\
\hline 矿粉30\% & 315 & 135 & 0 & 1350 & 225 \\
\hline $20 \%$ 矿粉, $10 \%$ 粉煤灰 & 315 & 90 & 45 & 1350 & 225 \\
\hline $10 \%$ 矿粉, $20 \%$ 粉煤灰 & 315 & 45 & 90 & 1350 & 225 \\
\hline 粉煤灰 $30 \%$ & 315 & 0 & 135 & 1350 & 225 \\
\hline $30 \%$ 矿粉, $10 \%$ 粉煤灰 & 270 & 135 & 45 & 1350 & 225 \\
\hline $20 \%$ 矿粉, $20 \%$ 粉煤灰 & 270 & 90 & 90 & 1350 & 225 \\
\hline $10 \%$ 矿粉, $30 \%$ 粉煤灰 & 270 & 45 & 135 & 1350 & 225 \\
\hline $40 \%$ 矿粉, $10 \%$ 粉煤灰 & 225 & 180 & 45 & 1350 & 225 \\
\hline $30 \%$ 矿粉, $20 \%$ 粉煤灰 & 225 & 135 & 90 & 1350 & 225 \\
\hline $20 \%$ 矿粉, $30 \%$ 粉煤灰 & 225 & 90 & 135 & 1350 & 225 \\
\hline $10 \%$ 矿粉, $40 \%$ 粉煤灰 & 225 & 45 & 180 & 1350 & 225 \\
\hline
\end{tabular}

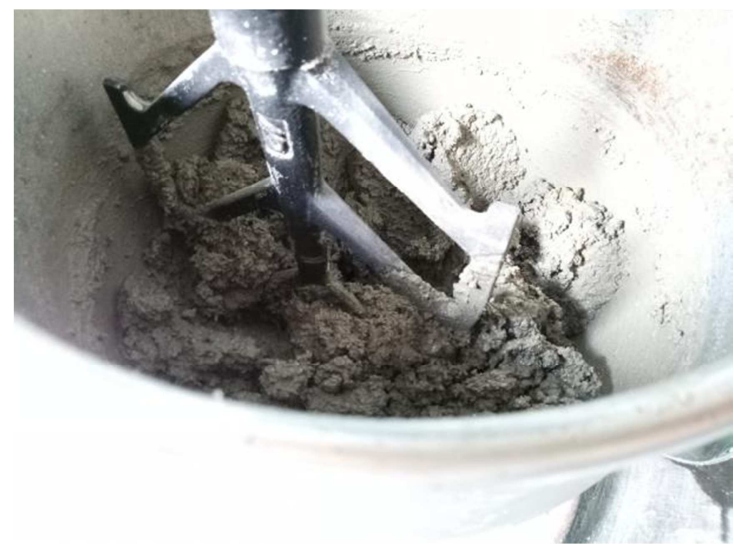

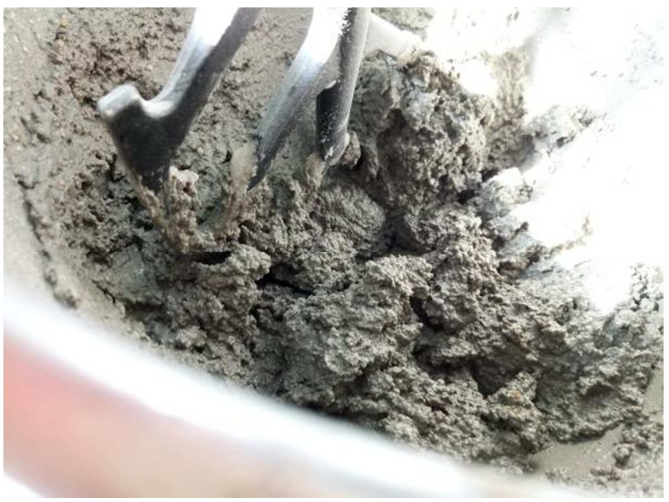

图1 胶砂摚拌状态。 


\section{2. 原材料性能}

水泥: P.O42.5水泥, $28 \mathrm{~d}$ 强度 $51.7 \mathrm{MPa}$, 细度 9.4 , 比 表面积 $325 \mathrm{~m} 2 / \mathrm{kg}$, 烧失量 3.7 。

粉煤灰：II级粉煤灰, 细度 13.3 , 烧失量 $5.5 \%$, 需水 量比 $98 \%$ 。

矿粉: S95 级矿粉, 比表面积 $432 \mathrm{~m} 2 / \mathrm{kg}$, 烧失量 $0.9 \%$, 7 天活性指数 $78 \%, 28$ 天活性指数 $98 \%$ 。
砂: 水泥胶砂试验用ISO标准砂。

\section{3. 流动度试验}

将材料按照 17 种组合按照 $\mathrm{GB} / \mathrm{T} 2419$ 水泥胶砂流动度 测定方法进行流动度试验, 取试验数据的平均值。试验结 果如下表:

表2 粉煤灰、矿粉单双掺胶砂流动度试验数据。

\begin{tabular}{|c|c|c|c|c|c|}
\hline 掺合料取代率 & 流动度 $(\mathrm{mm})$ & 掺合料取代率 & 流动度 (mm) & 掺合料取代率 & 流动度 $(\mathrm{mm})$ \\
\hline $0 \%$ & 212 & $30 \%$ 矿粉 & 215 & $10 \%$ 矿粉, $30 \%$ 粉煤灰 & 237 \\
\hline $10 \%$ 矿粉 & 219 & $20 \%$ 矿粉, $10 \%$ 粉煤灰 & 219 & $40 \%$ 矿粉, $10 \%$ 粉煤灰 & 237 \\
\hline $10 \%$ 粉煤灰 & 221 & $10 \%$ 矿粉, $20 \%$ 粉煤灰 & 221 & $30 \%$ 矿粉, $20 \%$ 粉煤灰 & 237 \\
\hline $20 \%$ 矿粉 & 213 & $30 \%$ 粉煤灰 & 230 & $20 \%$ 矿粉, $30 \%$ 粉煤灰 & 239 \\
\hline $10 \%$ 矿粉, $10 \%$ 粉煤灰 & 218 & $30 \%$ 矿粉, $10 \%$ 粉煤灰 & 236 & $10 \%$ 矿粉, $40 \%$ 粉煤灰 & 238 \\
\hline $20 \%$ 粉煤灰 & 225 & $20 \%$ 矿粉, $20 \%$ 粉煤灰 & 235 & & \\
\hline
\end{tabular}
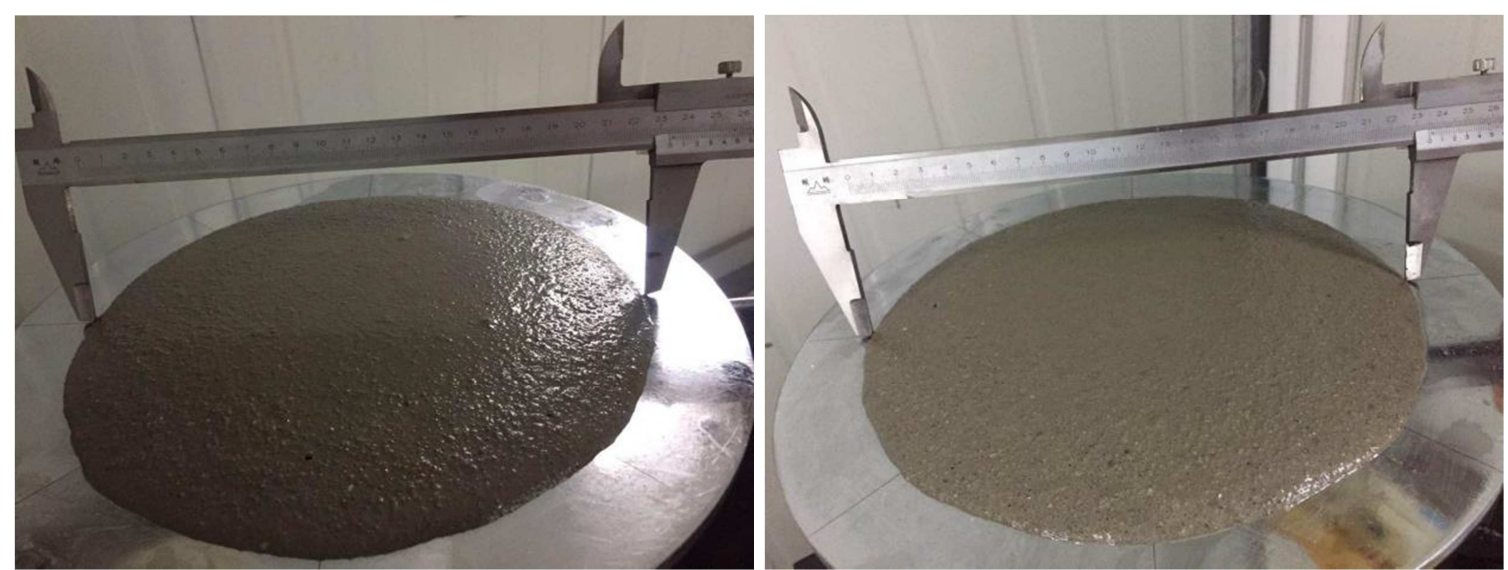

图2 流动度试验。

\section{不同取代率流动度趋势图}

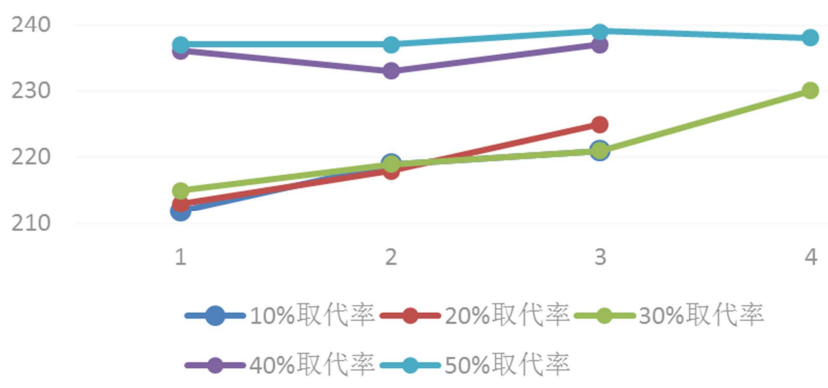

图3 不同取代率胶砂流动度趋势图（其中 $10 \%$ 数据线起点为基准水泥）。

通过上图流动度变化可以明显看出: 随着取代率的增 加流动度呈明显上升趋势; 同样的取代量时随着粉煤灰占 比的增加流动度呈上升趋势; 随着取代率的升高, 矿粉和 粉煤灰的占比不同对流动度的影响越来越小, 当取代率达 到 $50 \%$ 时, 流动度基本保持稳定不受材料占比影响。

这是由于粉煤灰和矿粉较水泥相比密度较小, 同质量 的胶材掺合料体积更大, 更有利于对骨料空隙的填充, 包 裹并润滑骨料颗粒, 可增加绿色混凝土流动性, 改善和易
性。粉煤灰的微集料效应和滚珠效应可以明显改善混凝土 工作性能, 特别是对大体积混凝土的施工。

\section{4. 强度试验}

17 种组合方式按照 GB/T 17671-1999水泥胶砂强度检 验方法进行胶砂强度试验, 取试验数据得平均值。试验结 果如下表: 
表3 粉煤灰、矿粉单双掺胶砂 $7 \mathrm{~d}$ 抗折强度数据。

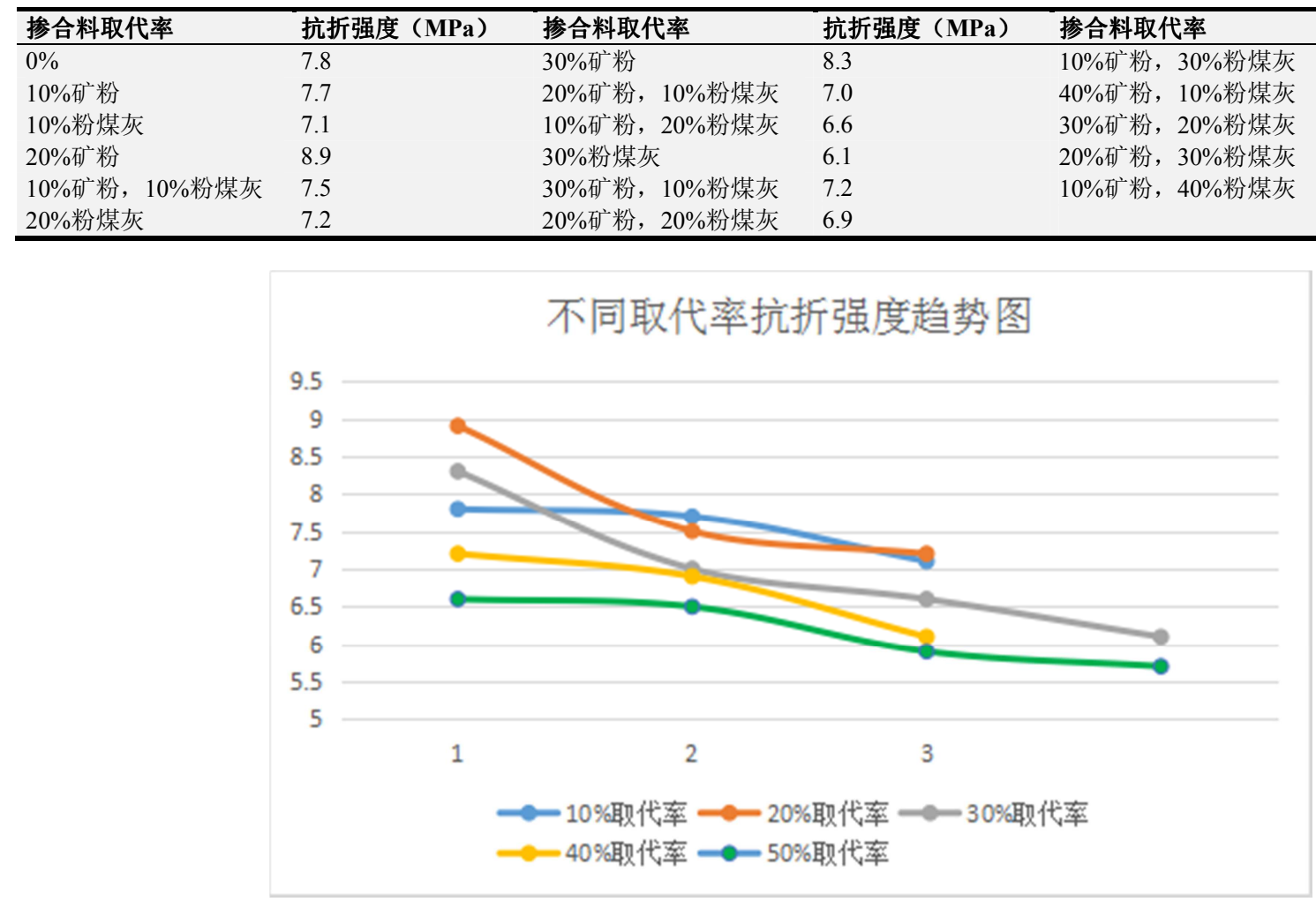

图4 不同取代率胶砂 $7 \mathrm{~d}$ 抗折强度趋势图（其中 $10 \%$ 数据线起点为基准水泥）。

表4 粉煤灰、矿粉单双掺胶砂 $7 \mathrm{~d}$ 抗压强度数据。

\begin{tabular}{|c|c|c|c|c|c|}
\hline 掺合料取代率 & 抗压强度（MPa） & 掺合料取代率 & 抗压强度（MPa） & 掺合料取代率 & 抗压强度（MPa） \\
\hline $0 \%$ & 37.8 & $30 \%$ 矿粉 & 36.5 & $10 \%$ 矿粉, $30 \%$ 粉煤灰 & 24.9 \\
\hline $10 \%$ 矿粉 & 37.6 & $20 \%$ 矿粉, $10 \%$ 粉煤灰 & 30.8 & $40 \%$ 矿粉, $10 \%$ 粉煤灰 & 27.2 \\
\hline $10 \%$ 粉煤灰 & 31.3 & $10 \%$ 矿粉, $20 \%$ 粉煤灰 & 27.2 & $30 \%$ 矿粉, $20 \%$ 粉煤灰 & 26.9 \\
\hline $20 \%$ 矿粉 & 37.0 & $30 \%$ 粉煤灰 & 24.4 & $20 \%$ 矿粉, $30 \%$ 粉煤灰 & 24.2 \\
\hline $10 \%$ 矿粉, $10 \%$ 粉煤灰 & 31.2 & $30 \%$ 矿粉, $10 \%$ 粉煤灰 & 28.0 & $10 \%$ 矿粉, $40 \%$ 粉煤灰 & 20.7 \\
\hline $20 \%$ 粉煤灰 & 28.3 & $20 \%$ 矿粉, $20 \%$ 粉煤灰 & 26.4 & & \\
\hline
\end{tabular}

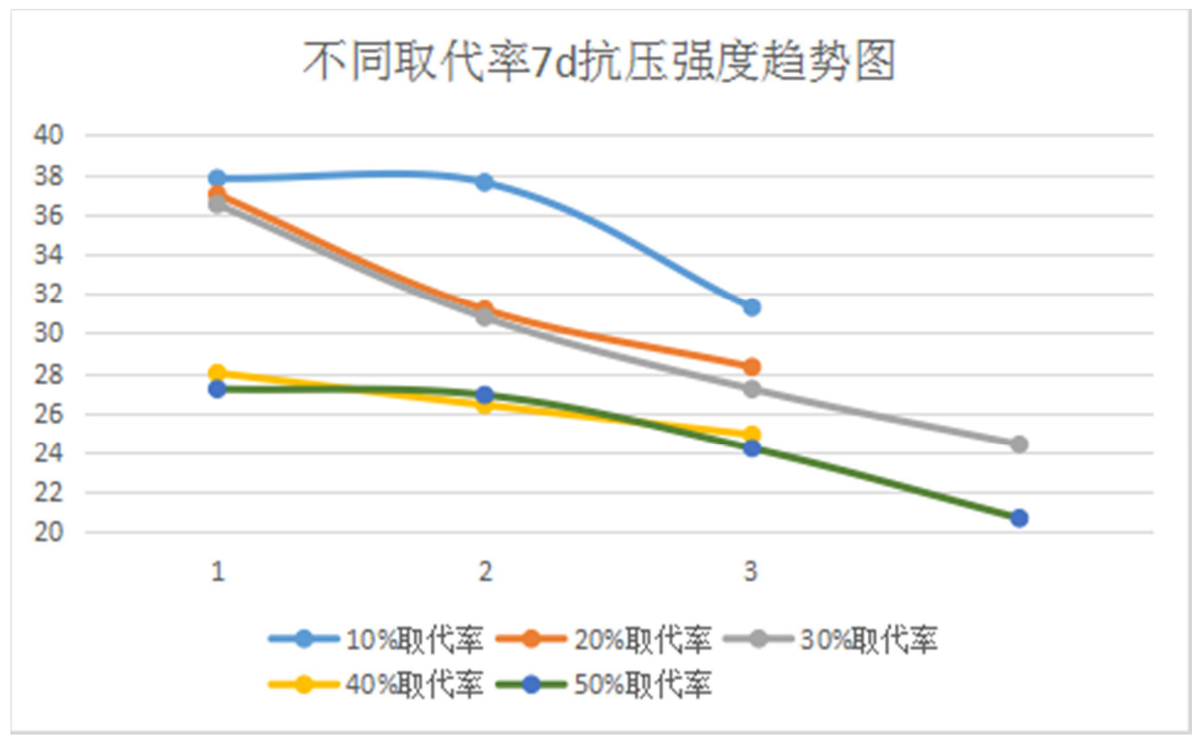

图5 不同取代率胶砂 $7 \mathrm{~d}$ 抗压强度趋势图（其中 $10 \%$ 数据线起点为基准水泥）。 
表5 矿粉掺量相同时随粉煤灰用量不同7d抗压强度数据。

\begin{tabular}{|c|c|c|c|c|c|c|c|c|c|}
\hline 取代率 & $\begin{array}{c}\text { 抗压强度 } \\
(\mathrm{MPa})\end{array}$ & 取代率 & $\begin{array}{c}\text { 抗压强度 } \\
(\mathrm{MPa})\end{array}$ & 取代率 & $\begin{array}{c}\text { 抗压强度 } \\
(\mathrm{MPa})\end{array}$ & 取代率 & $\begin{array}{c}\text { 抗压强度 } \\
(\mathrm{MPa})\end{array}$ & 取代率 & $\begin{array}{c}\text { 抗压强度 } \\
(\mathrm{MPa})\end{array}$ \\
\hline $0 \%$ & 37.8 & $10 \%$ 矿粉 & 37.6 & $20 \%$ 矿粉 & 37 & $30 \%$ 矿粉 & 36.5 & $0 \%$ & 37.8 \\
\hline $10 \%$ 矿粉 & 37.6 & $\begin{array}{l}10 \% \text { 矿粉 } 10 \% \\
\text { 粉煤灰 }\end{array}$ & 31.2 & $\begin{array}{l}20 \% \text { 矿粉 } \\
10 \% \text { 粉煤灰 }\end{array}$ & 30.8 & $\begin{array}{l}30 \% \text { 矿粉 } \\
10 \% \text { 粉煤灰 }\end{array}$ & 28.0 & $10 \%$ 粉煤灰 & 31.3 \\
\hline 20\%矿粉 & 37.0 & $\begin{array}{l}10 \% \text { 矿粉 } 20 \% \\
\text { 粉煤灰 }\end{array}$ & 27.2 & $\begin{array}{l}20 \% \text { 矿粉 } \\
20 \% \text { 粉煤灰 }\end{array}$ & 26.4 & $\begin{array}{l}30 \% \text { 矿粉 } \\
20 \% \text { 粉煤灰 }\end{array}$ & 26.9 & $20 \%$ 粉煤灰 & 28.3 \\
\hline $30 \%$ 矿粉 & 36.5 & $\begin{array}{l}10 \% \text { 矿粉 } 30 \% \\
\text { 粉煤灰 } \\
10 \% \text { 矿粉 } 40 \% \\
\text { 粉煤灰 }\end{array}$ & $\begin{array}{l}24.9 \\
20.7\end{array}$ & $\begin{array}{l}20 \% \text { 矿粉 } \\
30 \% \text { 粉煤灰 }\end{array}$ & 24.2 & & & $30 \%$ 粉煤灰 & 24.4 \\
\hline
\end{tabular}

\section{矿粉掺量相同时随粉煤灰用量不同7d抗压强度趋势}

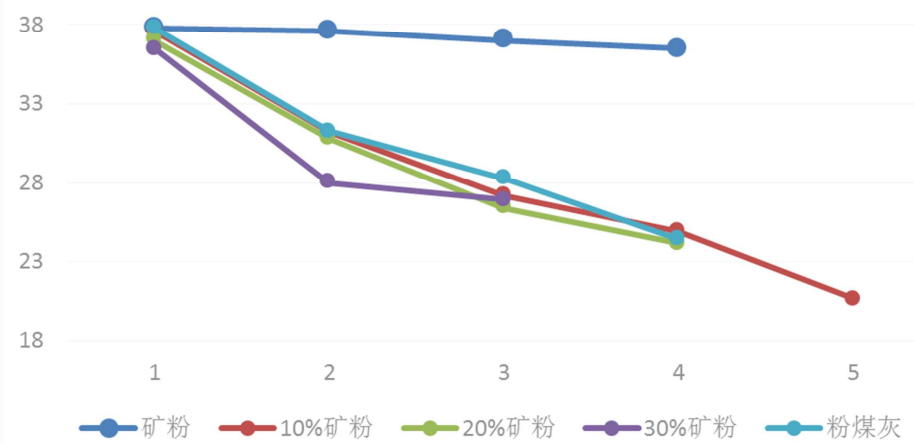

图6 相同矿粉掺量时随粉煤灰取代率不同7d抗压强度趋势图（其中10\%数据线起点为基准水泥）。

通过 $7 \mathrm{~d}$ 抗折和抗压强度数据的分析, 可以看出: 随着 掺合料取代率的增加抗折强度呈现先增后降的趋势, 早期 抗压强度呈下降趋势; 矿粉的活性较好，对胶砂早期强度 影响较小; 在粉煤灰掺量相同的情况下, 随着矿粉的增加,
强度变化不明显; 矿粉掺量相同的情况下，随着粉煤灰掺 量的增加早期抗压强度呈明显下降趋势。故可得出结论: 矿粉的掺加不影响粉煤灰在胶砂中强度的体现。

表6 28d抗折强度数据。

\begin{tabular}{llllll}
\hline 掺合料取代率 & 抗折强度 $(\mathbf{M P a})$ & 掺合料取代率 & 抗折强度 $(\mathbf{M P a})$ & 掺合料取代率 & 抗折强度 $(\mathbf{M P a})$ \\
\hline $0 \%$ & 10.6 & $30 \%$ 矿粉 & 10.3 & $10 \%$ 矿粉, $30 \%$ 粉煤灰 & 10.0 \\
$10 \%$ 矿粉 & 10.3 & $20 \%$ 矿粉, $10 \%$ 粉煤灰 & 9.8 & $40 \%$ 矿粉, $10 \%$ 粉煤灰 & 9.9 \\
$10 \%$ 粉煤灰 & 9.9 & $10 \%$ 矿粉, $20 \%$ 粉煤灰 & 10.2 & $30 \%$ 矿粉, $20 \%$ 粉煤灰 & 9.7 \\
$20 \%$ 矿粉 & 10.6 & $30 \%$ 粉煤灰 & 9.6 & $20 \%$ 矿粉, $30 \%$ 粉煤灰 & 9.5 \\
$10 \%$ 矿粉, $10 \%$ 粉煤灰 & 10.2 & $30 \%$ 矿粉, $10 \%$ 粉煤灰 & 10.9 & $10 \%$ 矿粉, $40 \%$ 粉煤灰 & 9.2 \\
$20 \%$ 粉煤灰 & 9.7 & $20 \%$ 矿粉, $20 \%$ 粉煤灰 & 10.3 & & \\
\hline
\end{tabular}

\section{不同取代率抗折强度趋势图}

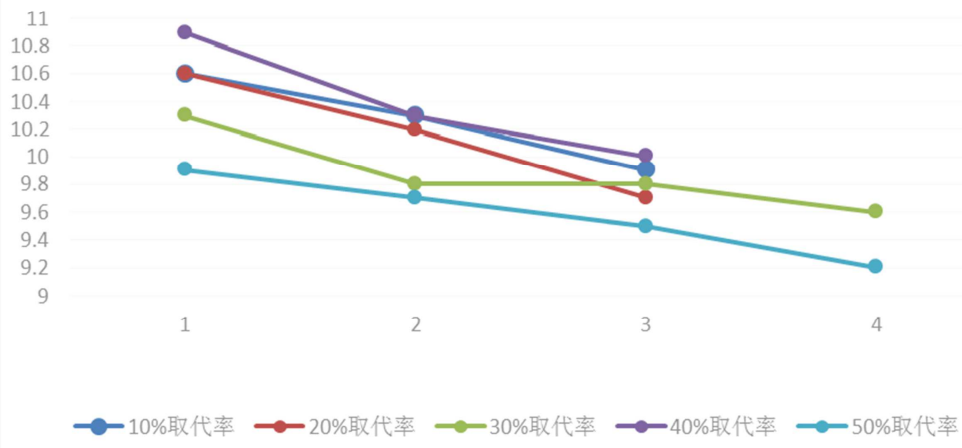

图7 不同取代率胶砂试块的 $28 \mathrm{~d}$ 抗折强度（其中 $10 \%$ 数据线起点为基准水泥）。 
表7 28d抗折强度数据。

\begin{tabular}{llllll}
\hline 掺合料取代率 & 抗压强度 $(\mathbf{M P a})$ & 掺合料取代率 & 抗压强度 $(\mathbf{M P a})$ & 掺合料取代率 & 抗压强度 $(\mathbf{M P a})$ \\
\hline $0 \%$ & 47.8 & $30 \%$ 矿粉 & 51.8 & $10 \%$ 矿粉, $30 \%$ 粉煤灰 & 44.6 \\
$10 \%$ 矿粉 & 47.6 & $20 \%$ 矿粉, $10 \%$ 粉煤灰 & 48.2 & $40 \%$ 矿粉, $10 \%$ 粉煤灰 & 48.4 \\
$10 \%$ 粉煤灰 & 44.7 & $10 \%$ 矿粉, $20 \%$ 粉煤灰 & 45.4 & $30 \%$ 矿粉, $20 \%$ 粉煤灰 & 44.2 \\
$20 \%$ 矿粉 & 51.6 & $30 \%$ 粉煤灰 & 37.4 & $20 \%$ 矿粉, $30 \%$ 粉煤灰 & 42.5 \\
$10 \%$ 矿粉, $10 \%$ 粉煤灰 & 48.9 & $30 \%$ 矿粉, $10 \%$ 粉煤灰 & 52.6 & $10 \%$ 矿粉, $40 \%$ 粉煤灰 & 38.3 \\
$20 \%$ 粉煤灰 & 45.5 & $20 \%$ 矿粉, $20 \%$ 粉煤灰 & 46.6 & & \\
\hline
\end{tabular}

\section{不同取代率抗压强度趋势图}

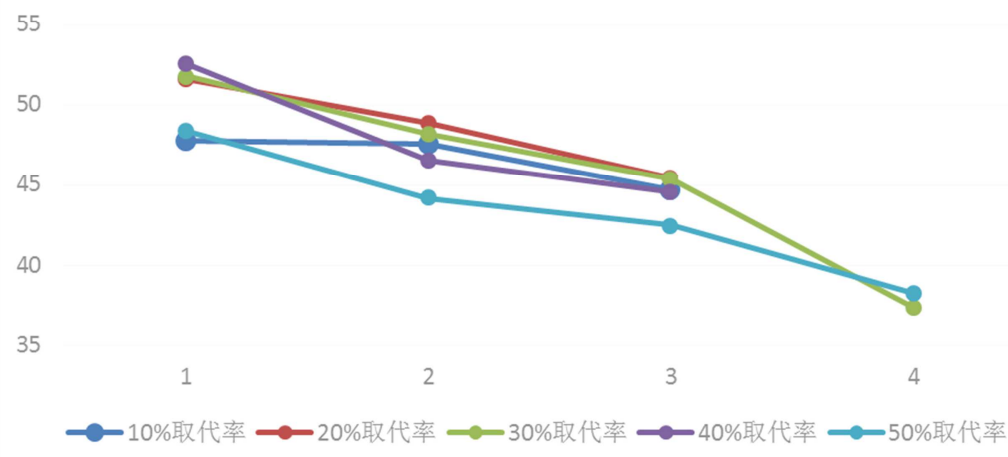

图8 胶砂试块的 $28 \mathrm{~d}$ 抗压强度（其中 $10 \%$ 数据线起点为基准水泥）。

表8 矿粉掺量相同时随粉煤灰用量不同28d抗压强度数据。

\begin{tabular}{|c|c|c|c|c|c|c|c|c|c|}
\hline 取代率 & $\begin{array}{c}\text { 抗压强度 } \\
(\mathrm{MPa})\end{array}$ & 取代率 & $\begin{array}{c}\text { 抗压强度 } \\
(\mathrm{MPa})\end{array}$ & 取代率 & $\begin{array}{c}\text { 抗压强度 } \\
(\mathrm{MPa})\end{array}$ & 取代率 & $\begin{array}{c}\text { 抗压强度 } \\
\text { (MPa) }\end{array}$ & 取代率 & $\begin{array}{c}\text { 抗压强度 } \\
(\mathrm{MPa})\end{array}$ \\
\hline $0 \%$ & 47.8 & $10 \%$ 矿粉 & 47.6 & \multirow{5}{*}{$\begin{array}{l}20 \% \text { 矿粉 } \\
20 \% \text { 矿粉 } \\
10 \% \text { 粉煤灰 } \\
20 \% \text { 矿粉 } \\
20 \% \text { 粉煤灰 } \\
20 \% \text { 矿粉 } \\
30 \% \text { 粉煤灰 }\end{array}$} & 51.6 & \multirow{5}{*}{$\begin{array}{l}30 \% \text { 矿粉 } \\
30 \% \text { 矿粉 } \\
10 \% \text { 粉煤灰 } \\
30 \% \text { 矿粉 } \\
20 \% \text { 粉煤灰 }\end{array}$} & 51.8 & $0 \%$ & 47.8 \\
\hline $10 \%$ 矿粉 & 47.6 & $\begin{array}{l}10 \% \text { 矿粉 } \\
10 \% \text { 粉煤灰 }\end{array}$ & 48.9 & & 48.2 & & 52.6 & $10 \%$ 粉煤灰 & 44.7 \\
\hline $20 \%$ 矿粉 & 51.6 & $\begin{array}{l}10 \% \text { 矿粉 } \\
20 \% \text { 粉煤灰 }\end{array}$ & 45.4 & & 46.6 & & 44.2 & $20 \%$ 粉煤灰 & 45.5 \\
\hline \multirow[t]{2}{*}{$30 \%$ 矿粉 } & \multirow[t]{2}{*}{51.8} & $\begin{array}{l}10 \% \text { 矿粉 } \\
30 \% \text { 粉煤灰 }\end{array}$ & 44.6 & & 42.5 & & & $30 \%$ 粉煤灰 & 37.4 \\
\hline & & $\begin{array}{l}10 \% \text { 矿粉 } \\
40 \% \text { 粉煤灰 }\end{array}$ & 38.3 & & & & & & \\
\hline
\end{tabular}

45

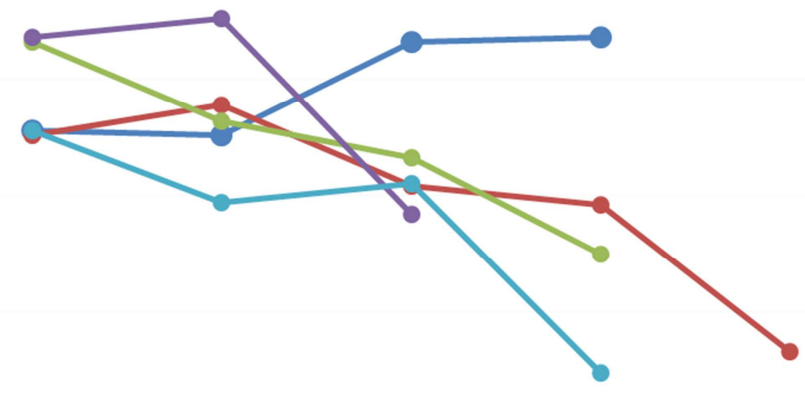

35

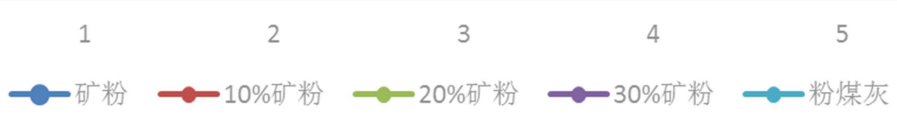

图9 矿粉掺量相同时随粉煤灰用量不同 $28 \mathrm{~d}$ d抗压强度趋势（其中 $10 \%$ 数据线起点为基准水泥）。 
通过对 $28 \mathrm{~d}$ 抗折和抗压强度数值的分析, 可以看出: 随 着掺合料用量的增加, 强度呈先升后降的趋势, 当取代率到 达 $40 \%$ 时强度最高, 其中又以矿粉占比较大时的强度更优。

通过在相同矿粉掺量前提下，调整不同粉煤灰用量时的 28 天抗压强度的分析可以看出: 随着矿粉替代率的增加胶砂 强度呈上升趋势, 相同矿粉取代率时随着粉煤灰取代率的提 高胶砂强度呈下降趋势。在矿粉取代率达到 $30 \%$ 时胶砂强度 最高, 同时加入 $10 \%$ 的粉煤灰, 使得强度达到峰值。

分析: 粉煤灰活性效应与矿粉活性相比较小, 此处体 现的应为粉煤灰微集料效应。粉煤灰中粒径很小的微珠和 颗粒, 在胶砂中可以相当于未水化的水泥颗粒, 极细小的 微珠相当于活泼的纳米材料, 能明显的改善和增强胶砂的 结构强度, 提高致密性。而且粉煤灰可稀释并反应其中的 氢氧化钠, 可使混凝土结构更密实。

\section{3. 结论}

在绿色混凝土生产中大量使用掺合料可以降低水泥 用量并改善混凝土性能。经试验结果分析, 随着复合掺合 料使用量的增加, 胶砂抗压强度呈现先升后降的趋势, 流 动性则随着使用量的增加持续增长。复合掺合料使用量达 到 $40 \%$ 时混凝土多项性能的改善最显著, 且不受矿物掺合 料占比的影响。在使用总掺量相同的情况下, 矿粉使用量 的增加对早期强度的改善明显优于粉煤灰。在复合使用掺 合料时，矿粉的大量使用 (30\%左右时) 不会对粉煤灰性 能的发挥造成影响, 并且随着矿粉使用量的增加, 粉煤灰 的细集料效应得到发挥, 更促进了粉煤灰活性的体现, 有 益于提高绿色混凝土的工作性能。

\section{4. 结语}

矿粉和粉煤灰已经成为了水泥混凝土中的重要组成 部分, 随着绿色混凝土的推广, 掺合料的使用范围和规模 将会越来越大。掺合料的使用可在一定范围内, 提高混凝
土的强度, 同时对改善混凝土的流动性、增加耐久性、降 低成本等方面均有明显的效果, 是绿色混凝土可持续发展 的重要方向。

\section{参考文献}

[1] JGJ 55-2011 普通混凝土配合比设计规程[S]. 北京: 中国 建筑工业出版社, 2011。

[2] 王坤.青岛地铁高性能衬砳混凝土试验研究[D]. 青岛理工 大学, 2010 。

[3] 朱效荣,李迁,张英男,等. 绿色高性能混凝土研究[M].2. 辽宁 大学出版社,2005。

[4] 马保国, 李永雓. 绿色高性能混凝土与矿物掺合料的研究 进展 [J]. 《武汉理工大学学报》, 1999, 21(5): 29-31。

[5] 王晓薇. 绿色高性能混凝土掺合料及性能的试验研究[D]. 燕山大学, 2008。

[6] 刘岩.石粉用作混凝土掺合料的初探 [J]. 《重庆建筑》.2010 年11期。

[7] 曹润武, 于琦. 大掺量粉煤灰和矿粉对混凝土强度的影响 [J]. 山西建筑, 2014, 40(2):121-124。

[8] 崔素萍,杜金. 矿物掺合料复合对再生粗骨料混凝土力学性 能的影响 [J].商品混凝土,2011年01期。

[9] 郑永宝,陈建功,刘东燕绿色高性能混凝土掺合料 (GC) 的 性能研究[J].《施工技术》,2003,32(4):20-21。

[10] 龚鄂川. 掺合料在绿色高性能混凝土中的应用综述 [J]. 江 西建材, 2017(2):11-12。 